\title{
Family Based Directly Observed Therapy on Culture Conversion in Newly Diagnosed Pulmonary Tuberculosis Patients
}

\author{
Duangjai Duangrithi ${ }^{1,2, *}$, Kamol Phojanamongkolkij ${ }^{3}$, Vipa Thanachartwet ${ }^{2}$, Varunee Desakorn ${ }^{2}$, \\ Yuthichai Kasetjaroen $^{4}$, Pasakorn Jitruckthai ${ }^{5}$, Duangjai Sahassananda ${ }^{6}$, Punnee Pitisuttithum ${ }^{2}$ \\ ${ }^{1}$ Department of Pharmacy Practice, Faculty of Pharmacy, Rangsit University, Pathumthani, Thailand \\ ${ }^{2}$ Department of Clinical Tropical Medicine, Faculty of Tropical Medicine, Mahidol University, Bangkok, Thailand \\ ${ }^{3}$ Queen Sawang Vadhana Memorial Hospital, Chon Buri, Thailand \\ ${ }^{4}$ Bureau of Tuberculosis, Department of Disease Control, Ministry of Public Health, Nonthaburi, Thailand \\ ${ }^{5}$ Chon Buri Hospital, Chon Buri, Thailand \\ ${ }^{6}$ Information Technology Unit, Faculty of Tropical Medicine, Mahidol University, Bangkok, Thailand \\ *Corresponding author: djdr@hotmail.com
}

Received January 26, 2014; Revised July 25, 2014; Accepted July 30, 2014

\begin{abstract}
Background: The effectiveness of directly observed therapy (DOT) on TB treatment outcome has been debated and different findings regarding different DOT practices and settings were demonstrated. Methodology: The observational study was conducted in new sputum smear and culture positive pulmonary TB patients at Queen Savang Vadhana Memorial Hospital and Chonburi Hospital, Chonburi province, Thailand during April 2010 and July 2012. The aim of this study was to evaluate the effect of family based DOT toward sputum culture conversion. Sputum AFB smear, culture and drug susceptibility test were performed at the time of diagnosis, the second and the fifth month of treatment. Baseline characteristic, clinical and laboratory parameters, treatment regimens and adverse events were recorded. Descriptive statistics were applied as appropriate. Results: Proportion of patients with illiteracy, visual alteration and hepatitis was significantly higher in family based DOT group ( $\mathrm{p}=0.026, \mathrm{p}=0.011$ and $\mathrm{p}=0.031$ ). Sputum smear and culture conversion at 2 and 5 months after treatment were not significantly different between family based DOT and self administer groups. Patients in both group achieved over $90 \%$ cure rate without significant difference $(\mathrm{p}=0.262)$. Conclusions: Continuing patient education, counseling and AEs monitoring should be incorporated into family based DOT to achieved successful outcome. DOT can be interfered by many factors. Therefore appropriate implementing of DOT can enhance effectiveness of DOT toward patient adherence and further achieve success in TB treatment and control.
\end{abstract}

Keywords: DOT, family base DOT, culture conversion, new pulmonary TB

Cite This Article: Duangjai Duangrithi, Kamol Phojanamongkolkij, Vipa Thanachartwet, Varunee Desakorn, Yuthichai Kasetjaroen, Pasakorn Jitruckthai, Duangjai Sahassananda, and Punnee Pitisuttithum, "Family Based Directly Observed Therapy on Culture Conversion in Newly Diagnosed Pulmonary Tuberculosis Patients." American Journal of Public Health Research, vol. 2, no. 4 (2014): 147-152. doi: 10.12691/ajphr-2-4-4.

\section{Introduction}

Irregular, interrupt or incomplete treatment of tuberculosis (TB) could result in drug resistance, treatment failure, relapse as well as transmission [1-3]. Furthermore, approximately $50 \%$ of TB patients had ever missed at least one anti-tuberculous dose [3]. Directly observed therapy (DOT) was the method for sustaining patient adherence to anti-tuberculous treatment by observing patients swallowing drugs [1,3] and it was recommended concomitantly with rifampicin containing regimen in 1980s in order to improve treatment outcome [3]. In 1990s, World Health Organization (WHO) incorporated DOT with standard short course regimen and named directly observed therapy, short course (DOTS) which was the key element of six principal components of The Stop TB Strategy [2].

DOT providers can be health care workers, community volunteers or family members [3]. DOT influencing treatment outcome of $\mathrm{TB}$ has been debated and different findings regarding different DOT practices and settings were demonstrated. Similar cure rates were reported between patients with DOT and self administration (SAT) [1]. Furthermore, lowest success rate was observed among patients provided DOT by health care worker $[1,4]$ whereas similar clinical outcomes were reported among patients provided DOT by clinic staffs, community health workers and families [1]. However, family based DOT showed potential advantage in cure rate compared to clinic based DOT [1]. In Thailand, family based DOT was more acceptable than layperson and volunteer [3]. 
Cure rate defined by sputum culture for Mycobacterium tuberculosis (40.8\%) and sputum acid fast bacilli (AFB) smear (32.4\%) among new definite pulmonary TB were significantly different $(\mathrm{p}<0.05)$ [5] and even none of patients with positive sputum AFB smear at the end of anti-tuberculous treatment were sputum culture positive for M. tuberculosis [6]. These may affect the benefit assessment of DOT toward treatment outcome.

To our knowledge, the association between family based DOT and sputum culture conversion among new definite cases of pulmonary TB was examined restrictedly. Therefore, this study aims to evaluate the effectiveness of family based DOT toward culture conversion rate of new pulmonary TB patients

\section{Materials and Methods}

\subsection{Study Design and Study Sites}

A prospective observational study was conducted at Queen Savang Vadhana Memorial Hospital and Chonburi Hospital in Chonburi province, Thailand during April 2010 and July 2012 in order to evaluate the effectiveness of family based DOT toward treatment outcome defined by sputum culture for $M$. tuberculosis in new pulmonary TB patients.

The inclusion criteria were: (1) patients with aged $\geq 14$ years; (2) patients having at least 2 or more of pulmonary or constitutional symptoms including cough, hemoptysis, chest pain or discomfort, dyspnea, fever, malaise, fatigue, weight loss and night sweat; (3) patients who were defined as pulmonary $\mathrm{TB}$ due to having abnormal chest compatible with pulmonary TB and having at least 1 of 3 sputum smear positive for AFB; and (4) patients who had first episode of TB or have taken anti-TB drugs for $<1$ month according to the 2010 WHO definition [7]. The exclusion criteria were: (1) patients having sputum culture negative for $M$. tuberculosis; and (2) patients having extrapulmonary TB without pulmonary TB.

Apart from education and counseling about TB and its treatment, DOT delivery was explained to all patients as well as their family members.

\subsection{Laboratories and Sputum Examinations}

Complete blood count, blood chemistries and HIV testing were performed at the time of diagnosis. All patients and their family members were educated about sputum collection procrdure. The sputum AFB smear was routinely performed at 2, 5 and 6 months after treatment at each hospital using Kinyoun method. Only one leftover sputum specimen with the highest positive AFB smear, prior to treatment, at 2 and 5 after treatment of each patient was sent to national TB reference laboratory center, Bangkok Thailand for mycobacterium culture as well as drug susceptibility test (DST) by conventional method using Löwenstein-Jensen solid media and automated liquid media culture systems (MGIT 960, BACTEC, USA). However, if patient was sputum AFB negative after treatment, one leftover sputum with the best quality specimen was sent for culture and DST.

Baseline characteristics, clinical and laboratory parameters as well as treatment regimens were collected and recorded in a pre-defined case record form. Adverse events (AEs) and treatment adherence were evaluated biweekly.

\subsection{Definitions}

Patients were classified into family based DOT group if DOT was delivered by their family members at least for the entire course of intensive phase according to Thai guideline for diagnosis and treatment of TB, otherwise were classified into SAT group. All patients as well as DOT observers were regularly supervised by telephone every 2 weeks. Treatment outcome was defined according to WHO Global Tuberculosis report 2012 [8]. Irregular adherence was identified if patients missed at least one dose of anti-tuberculous treatment according to Tuberculosis and Public Health, Policy and Principles in Tuberculosis Control [3].

\subsection{Sample Size Calculation}

The sample size was estimated based on the Thailand national TB program monitoring and evaluation report 2006-2007 which showed 77\% cure rate of new AFB smear positive patients with a 95\% confidence interval (CI) and the precision to be within $5 \%$ of the true value. A required sample size of at least 272 pulmonary TB patients was enrolled in our study.

\subsection{Statistical Analysis}

Data were analyzed using the statistical package SPSS for Windows version 18.0 (SPSS, Chicago, IL). Categorical variables were summarized as frequencies (percentages) and the comparison between 2 groups was performed by $\chi^{2}$ test. Numerical variables were tested for normality using the Kolmogorov-Smirnov test, if nonnormal distributed data, summarized as median (interquartile range; IQR) and 2 group comparisons was performed using Mann-Whitney $U$-test. The statistical significance was considered by two sided with $\mathrm{p}<0.05$.

\subsection{Ethical Consideration}

The study was approved by the ethics committee of faculty of Tropical Medicine, Mahidol University in Bangkok; and Queen Savang Vadhana Memorial Hospital and Chonburi Hospital in Chonburi province, Thailand. Written informed consent was obtained from all patients.

\section{Result}

\subsection{Baseline Characteristics}

Total of 233 new pulmonary TB patients were eligible for the study. Of these, 39 patients were treated under family based DOT and the rest were SAT. Baseline characteristics of patients in both groups were similar. Majority of patients in both groups were male (31/39, $79.5 \%$ VS 140/194, 72.2\%, p=0.456) with median age (IQR) of 40 years (31-51) and 37 year (28-50) in family based DOT and SAT group $(p=0.290)$. Over two third of patients in both group had history of smoking and alcohol consumption with the higher proportions in family based DOT group (30/39, 76.9\% VS 122/193, 63.2\%, p=0.145 and 29/39, 74.4\% VS 132/193, 68.4\%, p=0.585). Cough 
was the most common symptoms in both groups (36/39, 92.3\% VS 179/193, 92.7\%, p=1.000) and cavity on chest radiography was presented in one third of patients similarly in each group (11/32, 34.4\% VS 54/169, 32.0\%, $\mathrm{p}=0.950$ ). However, proportion of patients with illiteracy and low education in family based DOT group were significantly higher compared to SAT group (26/36, 72.2\% VS 90/179, 50.3\%, p=0.026) (Table 1).

\subsection{Sputum Conversions}

Smear conversion rate at the end of 2 and 5 months after treatment were not significantly higher in family based DOT group compared to SAT group (34/39, 87.2\% VS 162/192, 84.4\%, $p=0.841$ and 36/37, 97.3\% VS $177 / 185,95.7 \%, p=1.000)$. Similarly, culture conversion rate at the end of 2 and 5 month after treatment were not significantly higher in family based DOT group (29/38, 76.3\% VS 166/191, 86.9\%, p=0.153 and 35/37, 94.6\% VS 181/185, 97.8\%, $\mathrm{p}=0.262$ ).

Table 1. Baseline characteristics, clinical and laboratory parameters of among new pulmonary TB

\begin{tabular}{|c|c|c|c|c|c|c|}
\hline \multirow{2}{*}{ Characteristics } & \multirow{2}{*}{$\mathbf{n}$} & \multicolumn{2}{|r|}{ DOT } & \multicolumn{2}{|r|}{ Self administer } & \multirow{2}{*}{ P value } \\
\hline & & $\mathbf{n}$ & no(\%)/Median (IQR) & $\mathbf{n}$ & no(\%)/Median (IQR) & \\
\hline \multicolumn{7}{|l|}{ Baseline characteristics } \\
\hline Age & 233 & 39 & $40(31-51)$ & 194 & $37(28-50)$ & 0.290 \\
\hline Gender: male & 233 & 39 & $31(79.5)$ & 194 & $140(72.2)$ & 0.456 \\
\hline Married & 233 & 39 & $26(66.7)$ & 194 & $116(59.8)$ & 0.533 \\
\hline Education: illiterate or primary school & 215 & 36 & $26(72.2)$ & 179 & $90(50.3)$ & 0.026 \\
\hline Occupation: laborer & 233 & 39 & $18(46.2)$ & 194 & $106(54.6)$ & 0.428 \\
\hline Income $\leq 10,000 \mathrm{Baht}$ & 223 & 38 & $25(65.8)$ & 185 & $144(77.8)$ & 0.170 \\
\hline Smoking & 232 & 39 & $30(76.9)$ & 193 & $122(63.2)$ & 0.145 \\
\hline Alcoholic drinking & 232 & 39 & $29(74.4)$ & 193 & $132(68.4)$ & 0.585 \\
\hline BCG scar & 229 & 39 & $27(69.2)$ & 190 & $153(80.5)$ & 0.176 \\
\hline HIV & 225 & 37 & $4(10.8)$ & 188 & $21(11.2)$ & 1.000 \\
\hline Extrapulmonary TB & 233 & 39 & $2(5.1)$ & 194 & $11(5.7)$ & 1.000 \\
\hline \multicolumn{7}{|l|}{ Clinical characteristics } \\
\hline Cough & 232 & 39 & $36(92.3)$ & 193 & 179 (92.7) & 1.000 \\
\hline Fever & 232 & 39 & $24(61.5)$ & 193 & $141(43.1)$ & 0.210 \\
\hline Shortness of breath & 232 & 39 & $24(61.5)$ & 193 & $127(65.8)$ & 0.745 \\
\hline Fatigue & 232 & 39 & $23(59.0)$ & 193 & $99(51.3)$ & 0.484 \\
\hline Pleurisy & 232 & 39 & $19(48.7)$ & 193 & $105(54.4)$ & 0.636 \\
\hline Anorexia & 232 & 39 & $22(56.4)$ & 193 & $90(46.6)$ & 0.348 \\
\hline Night sweat & 232 & 39 & $18(46.2)$ & 193 & 77 (39.9) & 0.585 \\
\hline Hemoptysis & 232 & 39 & $17(43.6)$ & 193 & $61(31.6)$ & 0.208 \\
\hline Vomiting & 232 & 39 & $10(25.6)$ & 193 & $41(21.2)$ & 0.694 \\
\hline Nausea & 232 & 39 & $4(10.3)$ & 193 & $17(8.8)$ & 0.761 \\
\hline Cavity on CXR & 201 & 32 & $11(34.4)$ & 169 & $54(32.0)$ & 0.950 \\
\hline \multicolumn{7}{|l|}{ Physical examination } \\
\hline Body weight (kg) & 214 & 33 & $52.0(44.6-55.0)$ & 181 & $51.8(45.0-56.6)$ & 0.681 \\
\hline Body weight change (\%) & 204 & 31 & $-8.6(-15.2$ to -4.9$)$ & 173 & $-8.5(-14.9$ to -4.0$)$ & 0.634 \\
\hline Crepitation & 233 & 39 & $6(15.4)$ & 194 & $26(13.4)$ & 0.942 \\
\hline \multicolumn{7}{|l|}{ Laboratories } \\
\hline Hemoglobin (g/dL) & 143 & 28 & $11.4(10.0-13.0)$ & 115 & $11.5(9.7-13.2)$ & 0.827 \\
\hline Hematocrit (\%) & 143 & 28 & $35.2(28.2-38.5)$ & 115 & $34.4(30.3-39.3)$ & 0.770 \\
\hline White blood cell $\left(\mathrm{x} 10^{3} / \mathrm{mm}^{3}\right)$ & 143 & 28 & $10.0(7.7-14.2)$ & 115 & $10.4(8.2-13.5)$ & 0.867 \\
\hline Platelet $\left(\right.$ x $\left.10^{3} / \mathrm{mm}^{3}\right)$ & 143 & 28 & 386.5 (252.5-559.5) & 115 & $416.0(324.0-492.0)$ & 0.389 \\
\hline Sodium (mmol/l) & 91 & 21 & $136.0(131.5-137.5)$ & 70 & $134.0(130.0-136.1)$ & 0.216 \\
\hline BUN (mg/dl) & 107 & 25 & $10.6(7.5-13.0)$ & 82 & $9.0(7.0-12.0)$ & 0.264 \\
\hline Creatinine (mg/dl) & 118 & 26 & $0.8(0.6-0.8)$ & 92 & $0.8(0.7-0.9)$ & 0.290 \\
\hline Albumin (g/dl) & 156 & 26 & $3.3(3.0-3.8)$ & 130 & $3.3(2.9-3.7)$ & 0.786 \\
\hline Aspartate aminotransferase (U/l) & 160 & 29 & $28.0(21.0-37.5)$ & 131 & $25.0(19.0-43.0)$ & 0.569 \\
\hline Alanine aminotransferase (U/l) & 161 & 29 & $21.0(12.0-39.0)$ & 132 & $19.5(13.0-35.0)$ & 0.862 \\
\hline
\end{tabular}

There were no significant differences of drug resistances prior to treatment (2/38, 5.3\% VS 21/191, $11.0 \%, \mathrm{p}=0.384)$, at 2 months (0/38, 0 VS 5/190, 2.6\%, $\mathrm{p}=0.593)$ and 5 months (1/37, 2.7\% VS $2 / 184,1.1 \%$, $\mathrm{p}=0.424$ ) after treatment (Table 2). Primary and acquired MDR TB was found in 2 patients in SAT group.

\subsection{Treatment and Outcome}

Treatment and outcome were similar in both groups. Majority of patients in family based DOT and SAT groups were treated with the standard regimens according to WHO treatment of TB guideline 2010 in both intensive phase (37/39, 94.9\% VS 189/194, 97.4\%, p=0.332) and continuation phase (39/39, 100.0\% VS 192/194, 99.0\%, $\mathrm{p}=1.000$ ) with the similar median duration (IQR) of intensive [65.0 days (55.0-9.0) VS 62.5 (55.0-72.2), $\mathrm{p}=0.773$ ] and continuation phase [118.0 days (101.0-144.0) 
VS 120 days (112.0-145.0), $\mathrm{p}=0.523]$. Irregular adherence was not significantly different between family based DOT and SAT group, however it was observed more during continuation phase (16/39, 41.0\% VS 68/190, 35.8\%, $\mathrm{p}=0.663)$ than intensive phase $(7 / 39,17.9 \%$ VS $17 / 194$, $13.9 \%, p=0.688$ ). Cure rate was not significantly higher in family based DOT group compared to SAT group (28/39, 71.8\% VS 121/181, 66.9\%, p=0.682) (Table 3). Extrapulmonary TB was found in 1 patient from SAT group at 1 year after treatment.

Overall AEs were similar in both groups. During intensive phase, AEs were found equally in family based DOT and SAT groups (30/35, 85.7\% VS 143/168, 85.1\%, $\mathrm{p}=1.000$ ) and the incidence was lower in continuation phase (9/13, 69.2\% VS 54/70, 77.1\%, p=0.503) (Table 3). Arthralgia and skin reactions were the most common AEs during intensive [50.0\% (18/36) VS 52.1\% (87/167), $\mathrm{p}=0.965$ and $30.6 \%(11 / 36) \mathrm{VS} 47.9 \%(80 / 167), \mathrm{p}=0.087]$ and continuation phase [20.0\% (3/15) VS 39.7\% (27/68), $\mathrm{p}=0.254$ and $33.3 \%(5 / 15 /)$ VS $26.5 \%(18 / 68), p=0.751]$ similarly in both groups. However, proportion of patients with visual alteration and hepatitis were significantly higher in family based DOT group [12\% (20/167) VS $30.6 \%$ (11/36), $\mathrm{p}=0.011$ and $5.6 \%$ (2/36) VS 0, p=0.031]. On the other hand, numbness was not significantly higher in SAT groups [31.1\% (52/167) VS 13.9\% (5/36), $\mathrm{p}=0.060]$.

Table 2. Sputum examinations and drug resistances among patients in DOT and self administer groups

\begin{tabular}{|c|c|c|c|c|c|c|}
\hline \multirow{2}{*}{ Sputum examinations } & \multirow{2}{*}{$\mathbf{n}$} & \multicolumn{2}{|c|}{ DOT } & \multicolumn{2}{|c|}{ Self administer } & \multirow[b]{2}{*}{$P$ value } \\
\hline & & $\mathbf{n}$ & no (\%) & $\mathbf{n}$ & no (\%) & \\
\hline \multicolumn{7}{|l|}{ Smear negative } \\
\hline At 2 months & 231 & 39 & $34(87.2)$ & 192 & $162(84.4)$ & 0.841 \\
\hline At 5 months & 222 & 37 & 36 (97.3) & 185 & 177 (95.7) & 1.000 \\
\hline \multicolumn{7}{|l|}{ Culture negative } \\
\hline At 2 months & 229 & 38 & 29 (76.3) & 191 & $166(86.9)$ & 0.153 \\
\hline At 5 months & 222 & 37 & $35(94.6)$ & 185 & $181(97.8)$ & 0.262 \\
\hline \multicolumn{7}{|l|}{ Drug resistance } \\
\hline Prior to treatment & 229 & 38 & $2(5.3)$ & 191 & $21(11.0)$ & 0.384 \\
\hline At 2 months & 228 & 38 & 0 & 190 & $5(2.6)$ & 0.593 \\
\hline At 5 months & 221 & 37 & $1(2.7)$ & 184 & $2(1.1)$ & 0.424 \\
\hline
\end{tabular}

Table 3. Anti-tuberculous treatment and outcome among patients in DOT and self administer groups

\begin{tabular}{|c|c|c|c|c|c|c|}
\hline \multirow{2}{*}{ Characteristics } & \multirow{2}{*}{$\mathbf{n}$} & \multicolumn{2}{|r|}{ DOT } & \multicolumn{2}{|r|}{ Self administer } & \multirow[t]{2}{*}{ P value } \\
\hline & & $\mathbf{n}$ & no(\%)/Median (IQR) & $\mathbf{n}$ & no(\%)/Median (IQR) & \\
\hline Standard regimens & 233 & & & & & \\
\hline Intensive phase & & 39 & 37 (94.9) & 194 & $189(97.4)$ & 0.332 \\
\hline Continuous phase & & 39 & 39 (100.0) & 194 & 192(99.0) & 1.000 \\
\hline Duration of follow up & 179 & 33 & $289.0(262.0-342.5)$ & 146 & $287.0(267.0-336.0)$ & 0.773 \\
\hline \multicolumn{7}{|l|}{ Duration of treatment } \\
\hline Intensive phase & 233 & 39 & $65.0(55.0-90.0)$ & 194 & $62.5(55.0-72.2)$ & 0.288 \\
\hline Continuous phase & 232 & 39 & $118.0(101.0-144.0)$ & 193 & $120.0(112.0-145.0)$ & 0.523 \\
\hline \multicolumn{7}{|l|}{ Irregular adherence } \\
\hline Intensive phase & 233 & 39 & 7 (17.9) & 194 & 27(13.9) & 0.688 \\
\hline Continuous phase & 229 & 39 & $16(41.0)$ & 190 & $68(35.8)$ & 0.663 \\
\hline \multicolumn{7}{|l|}{ Missing days } \\
\hline Overall & 85 & 17 & $3.0(1.5-10.0)$ & 68 & $5.0(2.0-21.8)$ & 0.326 \\
\hline Intensive phase & 31 & 6 & $5.5(1.8-15.0)$ & 25 & $5.0(2.0-19.0)$ & 0.841 \\
\hline Continuous phase & 66 & 13 & $3.0(1.0-8.0)$ & 53 & $4.0(2.0-9.5)$ & 0.553 \\
\hline \multicolumn{7}{|l|}{ Adverse events } \\
\hline Intensive phase & 203 & 35 & $30(85.7)$ & 168 & $143(85.1)$ & 1.000 \\
\hline Continuous phase & 83 & 13 & $9(69.2)$ & 70 & $54(77.1)$ & 0.503 \\
\hline \multicolumn{7}{|l|}{ Outcome $^{\mathrm{a}}$} \\
\hline Cure & 233 & 39 & $28(71.8)$ & 181 & 121 (66.9) & 0.682 \\
\hline Default & 233 & 39 & $2(5.1)$ & 181 & $10(5.5)$ & 1.000 \\
\hline Success & 233 & 39 & 37 (94.9) & 181 & $164(90.6)$ & 0.538 \\
\hline
\end{tabular}

${ }^{\mathrm{a}}$ World Health Organization, Global tuberculosis report 2012.

\section{Discussion}

Factors influencing treatment adherence is multifactorial. Among TB patients, treatment adherence were affected by several factors e.g. socio-economic status, social support, accessible and patient friendly health systems, treatment duration, clinical improvement, adverse drug reactions, co-morbidities and the most importance, disease knowledge and its treatment [3, 9]. As comparing to other study in Bangkok, Thailand, most patients in family based DOT and SAT groups were illiterate (72.2\% and $50.3 \%$ VS $67.7 \%$ and $57.0 \%$ ), unemployed or laborer (46.2\% and $54.6 \%$ VS $53.0 \%$ and $54.7 \%$ ) and low income earning (65.8\% and $77.8 \%$ VS $81.3 \%$ and $87.8 \%$ ) [4]. Cavity on chest radiography was not significantly different between family based DOT and SAT groups (34.4\% VS 32.0\%) compared to study from northern Thailand (27.1\% VS 22.2\%) [10]. 
Although WHO recommends DOT for TB control; many studies showed no benefit of DOT over treatment outcomes [1,4,11-13]. This study supported those previous findings since culture conversion rates at 2 and 5 months after treatment between family based DOT and SAT groups were not significantly different. At the end of intensive phase, $87.2 \%$ and $84.4 \%$ of patients in family based DOT and SAT groups were sputum smear negative compared to $62 \%$ and $30 \%$ of the previous study [13]. The lower proportions from this previous study were due to missing data of sputum smear found in $15 \%$ and $46 \%$ of patients in family based DOT and SAT groups at the end of intensive phase [13]. Smear conversion rates at 2 and 5 months after treatment were slightly higher in family based DOT group than SAT group while culture conversion rates were slightly higher in SAT group. These could be explained by false positive sputum AFB smear after treatment caused by nonviable bacilli and probably misled to the effectiveness of DOT. However, culture conversion rates at 2 and 5 months after treatment in SAT group and family based DOT group were similar. Education was the most likely influencing factor since significantly higher education was observed in SAT group while the rest of factors were similar between both groups. It could be as effective as DOT toward treatment outcome. Therefore, knowledge of TB and its treatment should be intensively educated especially in patients with illiteracy or low educated as well as their family member. Then continuing education is strongly recommended.

Success rate in DOT group were not significant different to SAT group similarly to other findings $[1,4,10]$. Interestingly, cure rate in DOT group was $71.8 \%$ compared to $81-96 \%$ from previous study providing incentive [12]. Since most TB patients had low socioeconomic status, incentive such as transportation payment could be one of the strategies to achieve successful outcome. On the other hand, other previous study including relapse and failure cases showed the significantly different of treatment outcome among patients provided DOT and SAT with lower success rate compared to this study in both family based DOT (74.1\% VS 94.9\%) and SAT groups (77.4\% VS 90.6\%) [10]. Similarly, low cure rate by $34-76 \%$ and $41-67 \%$ among patients with family based DOT and SAT groups were reported from studies included retreatment cases as well as cases treated with non standard treatment regimens [1]. In addition, similar success rate was found in new pulmonary TB patients with DOT delivered by either health care workers or family members and SAT [13] indicating that DOT has no benefit for new TB cases [3]. However, the success rate obtained from this study was higher compared to latest national success rate of 85\% reported in 2010 [8]. This might be due to two important strategies using in this study. First, patients who could not attain clinic visit due to their work, prescription filled were provided after office hour and next appointment was arranged as soon as possible regarding patient convenience. Second, patients who missed doctors' appointments, they were immediately called and discussed for problems and their solutions.

Irregular adherence and default from treatment are the major causes of drug resistance and DOT has been proposed as the effective strategy by WHO and International Union Against Tuberculosis and Lung
Diseases [2,3]. However, similar treatment adherence, missing day and defaulted rate observed between both groups indicated that duration of DOT delivery modified from entire short course TB treatment as recommended by the stop TB strategies to at least in intensive phase as recommended by Thai guideline for diagnosis and treatment of TB may not be appropriate and might decrease effect of DOT over treatment outcome. Moreover, treatment adherence can be interfered by many factors as described previously [3,9]. Major cause of irregular adherence in both groups was alcoholic consumption similar to previous finding [3] while difficulty in asking sick leave and prioritized work over clinic visit were the major causes of missed appointment similar to previous studies [14]. Furthermore, significantly higher proportions of patients with AEs in DOT group could affect treatment adherence as reported previously [15]. Therefore, regular adherence assessment is necessary.

This study has some limitations. Recall bias is unavoidable for data obtaining by interview. Specimens after treatment were poor in quality because patients felt better and productive cough consequently subsided. This could influence isolation of $M$. tuberculosis, however it happens in the routine practice.

\section{Conclusion}

This study demonstrated that family based DOT had no effect on sputum culture conversion rate at 2 and 5 months after treatment as well as success rate. Since DOT delivery to all TB patients was unlikely in low income high burden TB countries like Thailand. Therefore, DOT should be focused on retreated or relapse cases while good quality TB service should be provided. Continuing patient education and counseling are fundamental necessity leading to understanding and co-operating in treatment. Emphasis on TB transmission among their families can increase their awareness and desire to cure of TB. Furthermore, TB affects predominately male in working active aged group and most of them were family leaders. Regular follow up should be feasible and accessible regarding patients working hours and cost for traveling. Fast tract services including direct clinic access and separated pharmacy service as well as patient friendly service should be established. In addition, treating TB needs the combination of several drugs with long duration so close monitoring for AEs should be performed. All these can enhance patient adherence and further achieve success in TB treatment and control.

\section{Acknowledgements}

We would like to thank our study participants for their co-operations. We also thank Dr. Pongsak Chunpongthong, Dr. Chureeratana Bowonwatanuwong, Dr. Ongard Kosintarajit, Dr. Apichart Chinnawan, Dr.Arun Lerdworawiwat, Dr. Poonlarb Panjaluk, Dr. Natcha Laopichainpong, nurses and staffs of TB clinic at Savang Vadhana Memorial Hospital and Chonburi Regional Hospital, Chonburi province, Thailand for participants enrollment; all staffs of Microbiology Unit at Savang Vadhana Memorial Hospital and Chonburi 
Regional Hospital, Chonburi province, Thailand for specimen handling and Mr. Somsak Rienthong, National TB Reference Laboratory, Bureau of Tuberculosis, Department of Disease Control, Ministry of Public Health, Nonthaburi, Thailand for Mycobacterium culture and sensitivity testing. Special thanks are extended to Assoc. Prof. Pratap Singhasivanon, Dean of the Faculty of Tropical Medicine and Dr. Arthit Ourairat President of Rangsit University for their support on this manuscript.

This study was supported by Faculty of Graduate Studies and Faculty of Tropical Medicine, Mahidol University, Bangkok, Thailand.

\section{Conflict of Interests}

The authors have no competing interests.

\section{List of Abbreviations}

$\begin{array}{ll}\text { AEs: } & \text { Adverse events } \\ \text { AFB: } & \text { acid fast bacilli } \\ \text { CI: } & \text { confidence interval } \\ \text { DOT: } & \text { directly observed therapy } \\ \text { DOTS: } & \text { directly observed therapy, short course } \\ \text { DST: } & \text { drug susceptibility test } \\ \text { IQR: } & \text { inter-quartile range } \\ \text { SAT: } & \text { self administration }\end{array}$

\section{References}

[1] Volmink J, Garner P, "Directly observed therapy for treating tuberculosis," Cochrane Database Syst Rev, CD003343, 2007.

[2] World Health Organization, The Stop TB Strategy Building on and enhancing DOTS to meet the TB-related Millennium Development Goals, 2006. 22p.
[3] Arnadottir T. Tuberculosis and Public Health. Policy and Principles in Tuberculosis Control, International Union Against Tuberculosis and Lung Disease, Paris, 2009, 547p.

[4] Okanurak K, Kitayaporn D, Wanarangsikul W, Koompong C, "Effectiveness of DOT for tuberculosis treatment outcomes: a prospective cohort study in Bangkok, Thailand," The International Journal of Tuberculosis and Lung Disease, 11(7).762-768. 2007.

[5] Migliori G, Ambrosetti M, Besozzi G, Casali L, Raviglione M, "Prospective multicentre study on the evaluation of antituberculosis treatment results in Italy: comparison of the culture- versus the smear-based methods," European Respiratory Journal, 13: 900-903. 1999.

[6] Vidal R, Martin-Casabona N, Juan A, Falgueras T, Miravitlles M, "Incidence and significance of acid-fast bacilli in sputum smears at the end of antituberculous treatment," CHEST, 109:1562-5. 1996.

[7] World Health Organization, Treatment of tuberculosis: guidelines, 4th ed.: World Health Organization, Geneva, 2010, 147p.

[8] World Health Organization, Global tuberculosis report, World Health Organization, Geneva, 2012, 272p.

[9] Aït-Khaled N, Alarcón E, Armengol R, et al, Management of Tuberculosis, A Guide to the Essentials of Good Practice, International Union Against Tuberculosis and Lung Disease, Paris, 2010, 104p.

[10] Prapanwonge A, Rattanajiemrungsri S, Klinchan S, Tipayamongkholgul $\mathrm{M}$, "Effects of DOT Observer for Tuberculosis Treatment in Mountainous Areas," Asia Journal of Public Health, 2 (3): 9-14. Sep2011.

[11] Cavalcante SC, Soares ECC, Pacheco AGF, Chaisson RE, Durovni B, Team DE, "Community DOT for tuberculosis in a Brazilian favela: comparison with a clinic model," The International Journal of Tuberculosis and Lung Disease 11 (5): 544-9. 2007.

[12] Ormerod LP, "Directly observed therapy (DOT) for tuberculosis: why, when, how and if?” Thorax, 54 Suppl 2: S42-5. 1999.

[13] Anuwatnonthakate A, Limsomboon P, Nateniyom S, et al. "Directly Observed Therapy and Improved Tuberculosis Treatment Outcomes in Thailand," PLoS ONE, 3, e3089. 2008

[14] Munro SA, Lewin SA, Smith HJ, Engel ME, Fretheim A, Volmink J, "Patient Adherence to Tuberculosis Treatment: A Systematic Review of Qualitative Research,” PLoS Med, 4, e238. 2007.

[15] Widjanarko B, Gompelman M, Dijkers M, van der Werf MJ, "Factors that influence treatment adherence of tuberculosis patients living in Java, Indonesia" Patient preference and adherence, 3, 231-238. 2009. 